\title{
Adaptación Argentina del Inventario de Fortalezas en Adolescentes (VIA- Youth): Propiedades Psicométricas y Alternativas para su Factorización
}

\author{
Argentinean Adaptation of the Values in Action Inventory of Strengths for Youth \\ (VIA-Youth): Psychometric Properties and Alternatives for its Factoring
}

María Julia Raimundi ${ }^{1}$, María Fernanda Molina ${ }^{2}$, Antonio Hernández-Mendo ${ }^{3}$ y Vanina Schmidt ${ }^{4}$

\section{Resumen}

El Values in Action Inventory of Strenghts for Youth (VIA-Youth) es un instrumento desarrollado para evaluar las fortalezas humanas en adolescentes. Este trabajo se propone presentar el proceso de adaptación de dicho instrumento para su uso con adolescentes de Argentina. En la primera etapa, se logró la adaptación lingüística y conceptual En la segunda etapa, se aplicó la versión local de la escala a una muestra de 542 adolescentes $($ varones $=45.40 \%$; mujeres $=54.60 \%)$, con una edad media de $14.92(D T=1.79)$ que asistían a escuelas públicas y privadas. Se estudió la capacidad de discriminación de los ítems y la fiabilidad. Con el objetivo de estudiar la validez de constructo se realizaron dos análisis factoriales exploratorios (AFE) mediante procedimientos diferentes. Se discuten los resultados a la luz de las recomendaciones actuales sobre AFE y la factorización encontrada para el uso del VIA-Youth en esta población de adolescentes.

Palabras clave: fortalezas humanas, adolescentes, inventario, adaptación, propiedades psicométricas

\begin{abstract}
The Values in Action Inventory of Strengths for Youth (VIA-Youth) is an instrument developed to evaluate adolescents' character strengths. The aim of this paper is to present the process of adaptation of this instrument for its use with adolescents from Buenos Aires, Argentina. In the first stage, linguistic and conceptual adaptation was achieved. In the second stage, the local version of the scale was applied to a sample of 542 adolescents (boys $=45.40 \%$; girls $=54.60 \%)$ with a mean age of $14.92(S D=1.79)$ attending to public schools and private. Discrimination capacity of items and reliability of subscales were studied. With the purpose of studying construct validity, two exploratory factor analyses (EFA) were performed using different procedures. The results are discussed in light of current recommendations for EFA and the factoring found for the use of the VIA-Youth in this population of adolescents.
\end{abstract}

Keywords: character strengths, adolescents, inventory, adaptation, psychometric properties

Agradecimientos: Se agradece a los alumnos, padres, docentes y autoridades de las escuelas que participaron en este estudio. A las Licenciadas Mariel Giménez, Lucía Bugallo, Yésica Vargas, María Sottani, Karina Mignolo, Celina Selva, Pamela Parma, Silvana Cataldi y a Nicolás Robles López por su colaboración en la recolección de datos y a Mariángeles Cossar por su colaboración con las traducciones de la escala.

\footnotetext{
${ }^{1}$ Dra. en Psicología (Universidad de Málaga/Universidad Nacional de La Plata). Becaria postdoctoral del Consejo Nacional de Investigaciones Científicas y Técnicas (CONICET). Instituto de Investigaciones, Facultad de Psicología, Universidad de Buenos Aires. Dirección postal: Av. Independencia 3065. Ciudad Autónoma de Buenos Aires. Argentina. Correo: jraimundi@psi.uba.ar

${ }^{2}$ Doctora en Psicología (Universidad Nacional de La Plata). Becaria postdoctoral del Consejo Nacional de Investigaciones Científicas y Técnicas (CONICET). Instituto de Investigaciones, Facultad de Psicología, Universidad de Buenos Aires. Dirección postal: Av. Independencia 3065. Ciudad Autónoma de Buenos Aires. Argentina. Correo: fernandamolina@psi.uba.ar

${ }^{3}$ Doctor en Psicología (Universidad de Santiago de Compostela). Profesor Titular del Dpto. Psicología Social, Antropología Social, Trabajo Social y Servicios Sociales. Facultad de Psicología, Universidad de Málaga. Dirección postal: Campus de Teatinos s/n. (29071) Málaga. España. Correo: mendo@uma.es

${ }^{4}$ Doctora en Psicología (Universidad de Buenos Aires). Investigadora Adjunta del Consejo Nacional de Investigaciones Científicas y Técnicas (CONICET). Instituto de Investigaciones, Facultad de Psicología, Universidad de Buenos Aires. Dirección postal: Av. Independencia 3065. Ciudad Autónoma de Buenos Aires. Argentina. Correo: vaninaschmidt@gmail.com
} 


\section{Introducción}

Entre los estudios de la Psicología, la entrada en el siglo XXI ha marcado el auge de propuestas teóricas que han compartido un objetivo común: el estudio del desarrollo positivo de las personas (Balaguer, Castillo, \& Duda, 2008). Muchos trabajos se han interesado por aquellas características individuales y de los entornos que promueven el bienestar y la satisfacción vital (e.g. Bassi, Steca, Monzani, Greco, \& Delle Fave, 2013). Este enfoque denominado "Psicología Positiva" se orienta hacia el estudio de las experiencias positivas subjetivas, las características individuales positivas y las instituciones que las posibilitan (Seligman \& Csikszentmihalyi, 2000).

Es en este contexto donde surge el estudio científico de las fortalezas humanas. El trabajo original de Peterson y Seligman (2004) se orientó a la revisión de los escritos tradicionales de los griegos, el Corán y otros textos que recogen virtudes o valores, desde aquellos más clásicos como los de Carlo Magno o Benjamin Franklin, a otros más actuales, como textos de John Templeton o el código de honor utilizado por los Boy Scouts, e incluyendo el análisis de diversos modelos psicológicos.

A partir de este trabajo, se concluyó que la mayoría de ellos valoraba seis virtudes fundamentales: la sabiduría y conocimiento, el valor, el amor y humanidad, la justicia, la templanza, y la espiritualidad y trascendencia. Estas virtudes podrían considerarse universales ya que han sido destacadas a lo largo de toda la historia de la humanidad (Peterson \& Seligman, 2004).

Debido a que el concepto de virtud es demasiado amplio, abstracto y poco práctico, se introduce el concepto de fortaleza para referirse a la manifestación psicológica de la virtud.

Las fortalezas son un conjunto de atributos positivos que se manifiestan en los pensamientos, sentimientos y conductas, que se vinculan con distintas situaciones de la vida y se desarrollan a lo largo del tiempo (Peterson \& Seligman, 2004).

Específicamente en la adolescencia, estas características positivas se asocian a diversos indicadores de bienestar y desarrollo saludable como satisfacción vital (Gillham et al., 2011; Park \& Peterson, 2006; Toner, Haslam, Robinson, \& Williams, 2012; Weber, Ruch, Littman-Ovadia, Lavy, \& Gai, 2013), rendimiento académico (Lounsbury, Fisher, Levy, \& Welsh, 2009; Park \& Peterson, 2006) y diversos intereses vocacionales (Proyer, Sidler, Weber, \& Ruch, 2012).

Si bien, no son muchos los estudios realizados con adolescentes de habla hispana, los resultados son prometedores en cuanto a las relaciones que establecen con factores protectores y promotores de la salud en esta etapa vital. Con una extensa muestra de adolescentes españoles, Giménez (2010) encontró, por un lado, relaciones positivas significativas entre las diferentes fortalezas y satisfacción con la vida, autoestima y afecto positivo, y por el otro, relaciones negativas con variables psicopatológicas como la depresión o el estrés. Las fortalezas que se repiten sistemáticamente asociadas a bienestar (gratitud, capacidad de amar, vitalidad y optimismo), son las mismas que se asocian de forma negativa con problemas psicopatológicos. Asimismo se encontró un papel mediacional de las fortalezas entre el clima familiar percibido y la satisfacción global con la vida. En un estudio longitudinal con adolescentes de Málaga, Ferragut et al. (2014) mostraron que las fortalezas se mantienen estables durante la adolescencia temprana (entre los 12 y los 14 años). En otro trabajo con la misma muestra de adolescentes se encontró una relación negativa entre las fortalezas y las creencias y actitudes sexistas, tanto en varones como en mujeres (Ferragut, Blanca, \& Ortiz-Tallo, 2013).

Estos trabajos con adolescentes españoles muestran el papel de las fortalezas y virtudes humanas como factores personales de gran relevancia para la salud y el desarrollo positivo en esta etapa vital.

\section{El Inventario de Fortalezas para adolescentes en su versión original}

El Values in Action Inventory of Strenghts for Youth (VIA-Youth, Park \& Peterson, 2006) se desarrolló basándose en la escala de adultos, diseñada por el mismo grupo de autores (Peterson \& Seligman, 2004). Aunque las fortalezas se desarrollan a lo largo de toda la vida, su manifestación en cada ciclo vital puede variar, por 
Tabla 1. Clasificación de virtudes y fortalezas humanas

\begin{tabular}{|c|c|c|c|c|c|}
\hline $\begin{array}{c}\text { Sabiduría y } \\
\text { conocimiento }\end{array}$ & Coraje & Humanidad & Justicia & Templanza & Trascendencia \\
\hline Creatividad & Valentía & $\begin{array}{l}\text { Capacidad } \\
\text { de amar }\end{array}$ & $\begin{array}{c}\text { Trabajo en } \\
\text { equipo }\end{array}$ & $\begin{array}{l}\text { Capacidad de } \\
\text { perdonar }\end{array}$ & $\begin{array}{l}\text { Apreciación } \\
\text { de la belleza y } \\
\text { excelencia }\end{array}$ \\
\hline Curiosidad & Perseverancia & Bondad & Equidad & Humildad & Gratitud \\
\hline Apertura mental & Integridad & $\begin{array}{l}\text { Inteligencia } \\
\text { social }\end{array}$ & Liderazgo & Prudencia & Optimismo \\
\hline $\begin{array}{l}\text { Amor por el } \\
\text { aprendizaje } \\
\text { Perspectiva }\end{array}$ & Vitalidad & & & Autorregulación & $\begin{array}{l}\text { Sentido del } \\
\text { humor } \\
\text { Espiritualidad }\end{array}$ \\
\hline
\end{tabular}

lo que se tuvieron en cuenta estas diferencias para desarrollar los ítems para la versión para niños y adolescentes (Park \& Peterson, 2003). A su vez, se realizaron grupos focales con adolescentes en 20 escuelas de los Estados Unidos para evaluar la pertinencia de la clasificación de las fortalezas para este grupo etario (ver Steen, Kachorek, \& Peterson, 2003 para más detalles).

El VIA-Youth evalúa las 24 fortalezas humanas surgidas de la clasificación de Peterson y Seligman (2004) (ver Tabla 1).

La versión original del cuestionario posee 198 ítems (Park \& Peterson, 2003, 2006). Cada una de las fortalezas consta de un total de siete a nueve ítems. Se han realizado adaptaciones, tanto de la versión para adultos (VIA-IS) como de la versión para adolescentes, en diferentes países como: España (Giménez, 2010), Japón (Otake et al., 2003 citado en Shimai, Otake, Park, Peterson, \& Seligman, 2006), Reino Unido (Linley et al., 2007), Croacia (Brdar \& Kashdan, 2010), Alemania (Ruch, Weber, Park, \& Peterson, 2014), Australia (Toner et al., 2012), Sudáfrica (Khumalo, Wissing, \& Temane, 2008; Van Eeden, Wissing, Dreyer, Park, \& Peterson, 2008) e Israel (Littman-Ovadia \& Lavy, 2012) indicando que se trata de un constructo relevante a nivel mundial. No obstante, aún queda mucho por avanzar en la operacionalización de las fortalezas, debido a que la estructura factorial del cuestionario no es clara y no se ha podido replicar en los diferentes estudios (Niemiec, 2013).

Respecto a esta problemática, Toner et al. (2012) postulan que la propuesta teórica de las seis virtudes no se corresponde con las actuales covariaciones de las fortalezas encontradas en los diferentes trabajos.

Hasta el momento, ninguno de los estudios anteriores citados que examinan el funcionamiento del VIA-IS y del VIA-Youth logra replicar la estructura de seis factores propuesta desde la teoría. En cambio, se encuentran evidencias de estructuras con tres y cuatro factores para la versión de adultos y de cuatro o cinco factores para la versión para adolescentes (Toner et al., 2012). A su vez, se encuentran diferencias en la composición de los factores encontrados.

Por otro lado, se desconoce si puede sostenerse una única estructura para adultos y para adolescentes (Toner et al., 2012). Específicamente para población adolescente, un estudio sugiere que la estructura del VIA-Youth es más homogénea o unidimensional que multidimensional (Van Eeden et al., 2008).

En la Tabla 2 se presentan las versiones del VIA-Youth publicadas hasta el momento con sus propiedades psicométricas.

En Argentina, Grinhauz y Castro Solano (2014) desarrollaron un instrumento para la evaluación de las fortalezas en niños: el Inventario de Virtudes y Fortalezas (IVyF). El mismo consta de 24 ítems bipolares (uno para cada fortaleza) con cinco opciones de respuesta que miden el grado en el que la persona se parece a la autodescripción enfrentada. Aun cuando el trabajo muestra que esta escala es una medida fiable, los autores de la escala original son escépticos frente a la viabilidad en la utilización de medidas demasiado breves, debido a que se trata de constructos complejos que necesitan una evaluación exhaustiva (Park \& Peterson, 2006). Es de suma importancia contar con instrumentos que permitan evaluar las fortalezas en su complejidad, logrando adaptaciones culturalmente adecuadas y transculturalmente comparables. Por lo tanto, el objetivo de este trabajo es presentar el proceso de adaptación del Cuestionario de Fortalezas para su uso con adolescentes de Argentina. 
Tabla 2. Versiones del VIA-Youth y sus propiedades psicométricas

\begin{tabular}{|c|c|c|c|c|c|}
\hline \multicolumn{2}{|c|}{ Muestra } & \multicolumn{3}{|c|}{ Propiedades psicométricas } & \multirow{3}{*}{ Autores } \\
\hline Grupo & Fdad & & idez & Fiahilidad & \\
\hline poblacional & Edad & Factorización & Otros tipos de validez & Flabilidad & \\
\hline
\end{tabular}

Preadolescentes y adolescentes $(n=$

Estados Unidos 306) entre 10 y 17 años.

Adolescentes $(n=$ 250).

\section{Alemania y Suiza (germano- parlantes) \\ Adolescentes $(n=$ 2110) entre $10 \mathrm{y}$ 17 años.}

Adolescentes $(n=$

Australia 501) entre 15 y 18 años.

ACP.

Cinco factores.

\section{ACP.} $(n=1049)$ entre 12 y 18 años.

Sudáfrica

Cinco factores

Análisis Factorial Confirmatorio (AFC). Ajuste pobre para el modelo de seis virtudes y

\section{Adolescentes $(n=1691)$ entre 13 y 17 años.}

Análisis de componentes principales (ACP) y Análisis Factorial Exploratorio (AFE) con máxima verosimilitud. Cuatro factores.

ACP.

Cinco factores. levemente mejor para modelo de un factor latente.

ACP. Un factor.
-Contenido: discusión en grupos focales y pruebas piloto.

-Convergente:

correlaciones con otros constructos,

correlaciones con

medidas externas

(medida del docente) y

con forma paralela de la escala.

-Otras evidencias de

validez de constructo: diferencias por género, edad y grupo sociocultural.

-Convergente: correlaciones con medidas externas (medida de los padres).

-Otras evidencias de validez de constructo: correlaciones con otros constructos, diferencias por género y edad.

-Otras evidencias de validez de constructo: correlaciones con otros constructos, diferencias por género.

-Otras evidencias de validez de constructo: correlaciones con otros constructos, diferencias por género y edad.

-Otras evidencias de validez de constructo: correlaciones con otros constructos.
-Consistencia interna: entre .66 y .87 (Park y Peterson, 2003) $\mathrm{y}$ entre .72 y .91 (Park y

Park y

Peterson

(2003,

Peterson, 2006). 2006)

-Test-retest (6 meses).

\section{-Consistencia interna: entre .60 y .87 , salvo autorregulación \\ Toner et \\ al. con .46 .

-Consistencia interna: entre .60 y .85 , salvo inteligencia social con .46 .

-Consistencia interna: entre .61 y .79, para todos los factores, a excepción de trabajo en equipo, solidaridad, autorregulación y humildad que va de .52 a .57 .

Van
Eeden et
al.
$(2008)$


Tabla 3. Características sociodemográficas de los adolescentes y sus familias según tipo de escuela

\begin{tabular}{|c|c|c|c|c|}
\hline \multicolumn{2}{|c|}{ Características } & $\begin{array}{l}\text { Escuelas } \\
\text { públicas } \\
(n=288) \\
\end{array}$ & $\begin{array}{l}\text { Escuelas } \\
\text { privadas } \\
(n=254) \\
\end{array}$ & $p$ \\
\hline \multicolumn{5}{|c|}{ Adolescente } \\
\hline Género & Mujeres $(n / \%)$ & $163(56.60)$ & $133(52.40 \%)$ & .323 \\
\hline Edad & $\begin{array}{l}M(D T) \\
\text { Rango }\end{array}$ & $\begin{array}{c}15.41(1.71) \\
12-19\end{array}$ & $\begin{array}{c}14.38(1.71) \\
11-18\end{array}$ & $<.001$ \\
\hline Repitencia & $\begin{array}{l}\text { No } \\
\text { Sí }\end{array}$ & $\begin{array}{c}206(72.00 \%) \\
80(28.00 \%)\end{array}$ & $\begin{array}{c}239(94.50 \%) \\
14(5.50 \%)\end{array}$ & $<.001$ \\
\hline \multicolumn{5}{|c|}{ Familia } \\
\hline $\begin{array}{l}\text { Composición familiar } \\
\%(n)\end{array}$ & $\begin{array}{c}\text { Monoparental } \\
\text { Biparental } \\
\text { Extendida } \\
\text { Compuesta } \\
\text { Otra }\end{array}$ & $\begin{array}{c}79(27.70 \%) \\
132(46.30 \%) \\
15(5.30 \%) \\
43(15.10 \%) \\
16(5.60 \%)\end{array}$ & $\begin{array}{c}49(19.50 \%) \\
165(5.70 \%) \\
11(4.40 \%) \\
16(6.40 \%) \\
10(4.00 \%)\end{array}$ & $<.001$ \\
\hline Personas en el hogar & $\begin{array}{c}M(D T) \\
\text { Rango }\end{array}$ & $\begin{array}{c}4.26(1.59) \\
1-14\end{array}$ & $\begin{array}{c}4.06(1.33) \\
2-11\end{array}$ & .119 \\
\hline \multicolumn{5}{|c|}{ Madre } \\
\hline $\begin{array}{l}\text { Nivel de instrucción } \\
\%(n)\end{array}$ & $\begin{array}{c}\text { Inferior secundario completo } \\
\text { Secundario completo } \\
\text { Terciario/Universitario } \\
\text { completo/en curso }\end{array}$ & $\begin{array}{l}132(47.00 \%) \\
57(20.30 \%) \\
70(24.90 \%)\end{array}$ & $\begin{array}{c}8(3.20 \%) \\
18(7.30 \%) \\
240(89.50 \%)\end{array}$ & $<.001$ \\
\hline $\begin{array}{l}\text { Situación laboral \% } \\
(n)\end{array}$ & $\begin{array}{c}\text { Empleo estable / regular } \\
\text { Desempleada } \\
\text { Ninguna } \\
\end{array}$ & $\begin{array}{l}187(69.80 \%) \\
47(17.50 \%) \\
34(12.70 \%) \\
\end{array}$ & $\begin{array}{c}213(87.70 \%) \\
14(5.80 \%) \\
16(6.60 \%) \\
\end{array}$ & $<.001$ \\
\hline \multicolumn{5}{|c|}{ Padre } \\
\hline $\begin{array}{l}\text { Nivel de instrucción } \\
\%(n)\end{array}$ & $\begin{array}{c}\text { Inferior a secundario completo } \\
\text { Secundario completo } \\
\text { Terciario/Universitario } \\
\text { completo/en curso }\end{array}$ & $\begin{array}{l}125(51.60 \%) \\
69(28.50 \%) \\
48(19.80 \%)\end{array}$ & $\begin{array}{c}12(5.00 \%) \\
25(10.40 \%) \\
203(84.60 \%)\end{array}$ & $<.001$ \\
\hline $\begin{array}{l}\text { Situación laboral \% } \\
\text { (n) }\end{array}$ & $\begin{array}{c}\text { Empleo estable / regular } \\
\text { Desempleado } \\
\text { Ninguna }\end{array}$ & $\begin{array}{c}190(81.20 \%) \\
18(7.70 \%) \\
26(11.10 \%)\end{array}$ & $\begin{array}{c}221(94.00 \%) \\
6(2.60 \%) \\
8(3.40 \%)\end{array}$ & $<.001$ \\
\hline
\end{tabular}

\section{Método}

\section{Participantes}

Adolescentes $(N=542)$ de entre 11 y 19 años de edad, de ambos géneros (54.60\% mujeres) que asistían a dos escuelas públicas (53.10\%) y dos privadas $(46.90 \%)$, todas ubicadas en la Ciudad Autónoma de Buenos Aires, Argentina (edad $M=14.92 ; D T=1.79$ ). En la Tabla 3 se presentan los datos sociodemográficos de los adolescentes.

Como puede observarse, los grupos no presentan diferencias en función del género, $x^{2}(1$, $542)=0.98, p=.323$, pero sí de la edad, $t(540)=$ $-6.95, p<.001$. Es decir, los adolescentes de las escuelas públicas son mayores que los adolescentes de las escuelas privadas. Esto es coherente con el hecho que se observa una mayor proporción de adolescentes que repitieron, al menos un curso, en las escuelas públicas que en las escuelas privadas, $x^{2}(1,539)=46.94, p<.001$. Por otro lado, se observan diferencias en el tipo de empleo de la madre, $x^{2}(2,511)=24.85, p<.001$, $\mathrm{y}$ el padre, $x^{2}(2,469)=17.87, p<.001$, y en el nivel de instrucción de la madre, $x^{2}(2,529)=226.13$, $p<.001$, y el padre, $x^{2}(2,482)=209.51, p<.001$. Es decir, en las escuelas privadas hay una mayor proporción de madres y padres con un empleo estable que en las escuelas públicas. En cuanto al nivel de instrucción del padre y la madre, en las escuelas privadas hay una mayor proporción de padres con un nivel de instrucción terciario o universitario completo o en curso, mientras que en las escuelas públicas es mayor la proporción de padres con un nivel educativo inferior al de 
secundario completo. También se observan diferencias en cuanto a la composición familiar, $x^{2}$ $(4,536)=22.99, p<.001$. En las escuelas privadas se observa una mayor proporción de familias biparentales, mientras que en las escuelas públicas se observa una mayor proporción de familias monoparentales y compuestas. No hay diferencias entre ambos grupos en el número de personas que conviven con el adolescente, $t(494,63)=-1.56$, $p=.119$.

Por lo tanto, se trata de una muestra de participantes de dos contextos escolares con diferencias esperables entre ellos en cuanto a las características del contexto familiar y la repetición en los grados escolares.

\section{Instrumentos}

Cuestionario socio-demográfico y sociofamiliar. Construido ad hoc para obtener datos acerca de las características sociodemográficas y familiares del adolescente.

Cuestionario de Fortalezas para adolescentes (VIA-Youth, Park \& Peterson, 2006). La versión de la escala resultante de la primera etapa de la adaptación poseía 202 ítems, de los cuales 198 son los originales y cuatro fueron creados para esta adaptación. El formato de respuesta es con una escala de tipo Likert de cinco puntos (desde muy parecido a mí hasta nada parecido a mî). En la consigna se le solicita al participante que responda en qué grado cada frase lo representa. Los ítems son del tipo "Creo que tengo el coraje o la valentía para enfrentar situaciones difíciles" (Valentía) y "Me entusiasmo cuando aprendo algo nuevo" (Amor al conocimiento y aprendizaje).

Perfil de Autopercepción para Adolescentes (SPPA, Harter, 1988; adaptación: Facio, Resett, Braude, \& Benedetto, 2006). Evalúa el autoconcepto a través de ocho dominios específicos: Competencias académicas, Aceptación social, Competencias deportivas, Apariencia física, Buen comportamiento, Atractivo amoroso, Competencia laboral $y$ Amistad íntima. A su vez evalúa la autoestima global. Cada subescala se compone de cinco ítems con cuatro opciones de respuesta. La escala SPPA ha sido aplicada en el presente estudio con el fin de obtener datos adicionales a favor de la validez de constructo. En la presente muestra se encontraron adecuados índices de consistencia interna (alfa de Cronbach): competencias académicas $=.62 ;$ aceptación social $=.68$; competencias deportivas $=.86$; apariencia física $=$ .92 ; competencia laboral $=.78$; atractivo amoroso $=.70$; buen comportamiento $=.70 ;$ amistad íntima $=.77$; autoestima global $=.77$.

\section{Procedimiento}

El presente trabajo cuenta con la aprobación de la Comisión de Evaluación de Conductas Responsables en Investigación (Facultad de Psicología, Universidad de Buenos Aires).

En primer lugar, se realizó la retro-traducción de la escala (inglés-español y español-inglés) por tres traductores independientes. La consigna para la versión adaptada fue diseñada tomando la consigna propuesta por los autores de la versión original de la escala (Park \& Peterson, 2006) pero también, considerando la versión española (Giménez, 2010).

La escala fue evaluada por tres jueces expertos respecto del grado de ajuste ítemconstructo, la adecuación sintáctica y semántica y la adecuación para el grupo etario al que está destinada. La versión española se tomó en cuenta para conocer en qué grado la versión argentina se asemejaba a ella.

Luego, se realizó una prueba piloto con la escala resultante de la evaluación de los jueces, con 10 adolescentes de diferentes edades con el objetivo de evaluar la compresión de la consigna y los ítems del instrumento. Es decir, se integraron las dos voces que participaron en la adaptación (jueces expertos y población diana) que constituyen fuentes independientes para garantizar la adecuación conceptual, cultural y lingüística del cuestionario en nuestro contexto.

En una segunda etapa, se contactó a las autoridades de las escuelas, se les explicó los objetivos de la investigación, y se obtuvo su permiso para la realización del estudio. Las escalas fueron administradas de forma grupal por evaluadores entrenados. Los adolescentes participaron en forma voluntaria y anónima, con consentimiento escrito por parte de los padres.

\section{Análisis de datos}

En primer lugar, se imputaron los datos perdidos, mediante el procedimiento de tendencia lineal en el punto, cuando no excedían el $30 \%$ de 
las respuestas del sujeto. Cuando el porcentaje de casos perdidos era mayor a este porcentaje, se eliminó el caso completo.

Para analizar el funcionamiento de los ítems se analizó su capacidad de discriminación por medio del análisis de la frecuencia de respuesta y las correlaciones ítem-total corregidas con el puntaje total de la escala a la que pertenecen. En las versiones previas del cuestionario (e.g. norteamericana y española) se asumió que los ítems de cada fortaleza se agrupaban entre sí para medir una única variable y a partir de ello se realizaron análisis factoriales para estudiar cómo se agrupaban las fortalezas en distintos factores. En este caso, se asume que ese parcel (cada fortaleza) mide lo que se pretende, pero no hay pruebas empíricas de ello. Según Arias (2008) un parcel es una puntuación total de un conjunto de ítems homogéneos y una condición imprescindible para su correcta utilización es que sean unidimensionales, es decir, es necesario demostrar que miden un solo constructo. Por lo tanto, para analizar la pertenencia de los ítems a cada una de las fortalezas, en primer lugar, se realizó un AFE sobre los ítems que pertenecían a cada escala, según la versión original, para analizar si los mismos se agrupaban en un mismo factor. En segundo lugar, se realizó un AFC con el objetivo de confirmar la estructura de cada fortaleza, en donde los ítems debían converger en un único factor global. En función de los análisis mencionados se seleccionaron los mejores ítems para componer la versión definitiva del instrumento. Se buscó encontrar una cantidad mínima de ítems con una alta validez y consistencia para medir el constructo. Para esto se tuvieron en cuenta los siguientes criterios: la capacidad de discriminación de los ítems, que los ítems presentaran una buena saturación en el AFE y AFC, que contribuyeran a la consistencia interna de la subescala a la que pertenecían, y que la versión adaptada se mantuviera lo más similar posible a la escala original.

Para analizar la estructura interna del instrumento, se realizó un AFE con el objetivo de reducir la cantidad de factores, de la misma manera que realizaron los autores de la versión original y la adaptación española. Se introdujeron como variables, los puntajes de las escalas (fortalezas) surgidas de los análisis anteriores. Se utilizó el índice de adecuación de la muestra de Kaiser-Meyer-Olkin, el determinante de la matriz y la prueba de esfericidad de Barttlet como requisito previo a la aplicación del análisis factorial.

Tabla 4. Estadísticos descriptivos de las fortalezas humanas evaluadas

\begin{tabular}{lcccccccc}
\hline Fortalezas & $M$ & $D T$ & Asimetría & Curtosis & Mínimo & Máximo & $\alpha$ & IC $\alpha$ \\
\hline Creatividad & 22.41 & 4.90 & -0.54 & -0.08 & 7 & 30 & .77 & {$[.74, .80]$} \\
Curiosidad & 23.71 & 4.61 & -0.67 & 0.06 & 8 & 30 & .74 & {$[.70, .77]$} \\
Perspectiva & 14.66 & 3.12 & -0.46 & 0.18 & 4 & 20 & .64 & {$[.59, .69]$} \\
Valentía & 23.03 & 4.28 & -0.55 & -0.02 & 8 & 30 & .72 & {$[.68, .76]$} \\
Apertura mental & 11.83 & 2.60 & -0.80 & 0.15 & 3 & 15 & .65 & {$[.60, .70]$} \\
Amor al aprendizaje & 19.49 & 4.01 & -0.63 & -0.13 & 5 & 25 & .77 & {$[.74, .80]$} \\
Perseverancia & 18.53 & 3.63 & -0.47 & -0.08 & 6 & 25 & .69 & {$[.65, .73]$} \\
Integridad & 12.41 & 3.75 & -0.08 & -0.65 & 4 & 20 & .75 & {$[.71, .78]$} \\
Vitalidad & 19.31 & 3.82 & -0.63 & 0.29 & 5 & 25 & .72 & {$[.68, .76]$} \\
Capacidad de amar & 15.52 & 3.38 & -0.87 & 0.33 & 4 & 20 & .65 & {$[.60, .70]$} \\
Solidaridad & 20.26 & 3.59 & -0.76 & 0.38 & 5 & 25 & .75 & {$[.72, .78]$} \\
Trab. en equipo & 23.81 & 4.12 & -0.85 & 1.08 & 6 & 30 & .76 & {$[.73, .79]$} \\
Equidad & 22.00 & 4.63 & -0.33 & -0.42 & 9 & 30 & .71 & {$[.67, .75]$} \\
Liderazgo & 18.93 & 4.71 & -0.06 & -0.36 & 6 & 30 & .75 & {$[.72, .78]$} \\
Capacidad de perdonar & 14.72 & 3.81 & -0.71 & 0.06 & 4 & 20 & .78 & {$[.75, .81]$} \\
Humildad & 26.79 & 4.87 & -0.49 & 0.10 & 9 & 35 & .66 & {$[.61, .70]$} \\
Prudencia & 6.98 & 2.75 & 0.51 & -0.39 & 3 & 15 & .62 & {$[.56, .67]$} \\
Autorregulación & 17.51 & 5.68 & -0.03 & -0.80 & 6 & 30 & .75 & {$[.72, .78]$} \\
Apreciación de la belleza & 18.15 & 4.97 & -0.51 & -0.41 & 5 & 25 & .77 & {$[.74, .80]$} \\
Gratitud & 17.44 & 2.40 & -1.19 & 1.61 & 8 & 20 & .55 & {$[.48, .61]$} \\
Optimismo & 19.47 & 4.20 & -0.86 & 0.69 & 5 & 25 & .78 & {$[.75, .81]$} \\
Sentido del humor & 15.81 & 3.47 & -0.84 & 0.57 & 4 & 20 & .82 & {$[.79, .84]$} \\
Religiosidad & 21.88 & 6.52 & -0.74 & -0.31 & 6 & 30 & .88 & {$[.86, .90]$}
\end{tabular}

Nota: IC = Intervalo de Confianza. 
Se realizaron dos AFE, con diferentes métodos. En primer lugar, con el programa SPSS se realizó mediante el método de máxima verosimilitud con rotación Oblimin con Kaiser. En segundo lugar, se realizó un AFE con el programa FACTOR 9.3 (Lorenzo-Seva \& Ferrando, 2015) siguiendo las recomendaciones actuales para realizar AFE (Ferrando \& LorenzoSeva, 2014; Lloret-Segura, Ferreres-Traver, Hernández-Baeza, \& Tomás-Marco, 2014)

Asimismo, se estudió la fiabilidad por medio del análisis de consistencia interna (alfa de Cronbach) de cada una de las escalas

Finalmente, con el objetivo de obtener evidencias adicionales a la validez de constructo se realizaron pruebas $t$ para muestras independientes para estudiar las fortalezas en función del género, edad y tipo de escuela. Para estudiar el tamaño del efecto se utilizó el coeficiente de correlación ( $r$ de Pearson), tomando los criterios de Cohen (1992) para su interpretación $($ bajo $=.10 ;$ mediano $=.30 ;$ alto $=$ $.50)$. Asimismo se realizaron correlaciones con medidas externas: dimensiones del autoconcepto y autoestima global.

Por lo tanto, en función de los análisis mencionados, los objetivos específicos de este trabajo son: explorar la estructura factorial del VIA-Youth al ser utilizado en adolescentes de población argentina, estudiar la validez de contenido, de constructo y la fiabilidad de la versión adaptada y comparar los resultados alcanzados respecto de la estructura factorial al utilizar AFE con la matriz de correlaciones de Pearson (mediante el SPSS) y el AFE con la matriz policórica (con el FACTOR).

\section{Resultados}

\section{Validez de contenido}

A partir de la evaluación de los jueces expertos y la administración de la escala a adolescentes de diferentes edades, se modificaron algunas expresiones para que fueran apropiadas para los adolescentes de nuestra cultura (e.g. se modificó la expresión "bromeo" por "hago chistes") y para el nivel de comprensión del grupo etario al que está destinada la escala (e.g. se modificó "presumo" por "no me la creo"").

Asimismo se crearon cuatro nuevos ítems para la fortaleza de Valentía, ya que los jueces sugirieron que la definición de esta fortaleza incluía aspectos no considerados en los reactivos de la escala original. La definición considera el "no echarse atrás por el miedo, los cambios, las dificultades o el dolor; defender lo que es justo a pesar de la oposición y actuar de acuerdo a las convicciones aunque sean impopulares". Los ítems sólo consideraban la valentía en situaciones sociales y de defensa de lo justo, por lo que se agregaron ítems acerca de la valentía en situaciones difíciles y la autopercepción sobre la valentía

De este modo se logró adaptar lingüística y conceptualmente el instrumento, obteniéndose una primera versión local.

\section{Capacidad de discriminación de los ítems}

Se conservaron aquellos ítems con una frecuencia de no más del $80.00 \%$ de las respuestas acumuladas en uno de los extremos (muy parecido a mí y algo parecido a mí o muy diferente a mí y algo diferente a mí). Se realizaron algunas excepciones por el valor teórico del ítem, sobre todo en la escala de Gratitud. Por otro lado, todos los ítems presentaron correlaciones ítem-total corregidas mayores a .30, indicando que todos los ítems conservados poseen una adecuada capacidad de discriminación.

En general, se observó que los puntajes medios de cada fortaleza muestran un sesgo positivo (ver Tabla 4) aunque se sostiene la variabilidad entre las respuestas de los participantes.

\section{Validez de constructo}

En primer lugar, los AFE realizados para cada una de las fortalezas mostraron que los ítems se agrupan de acuerdo a la fortaleza hipotetizada presentando un solo factor sin forzar en la mayoría de los casos. En aquellos casos (6) en donde resultaron dos factores se eliminaron aquellos ítems que se agrupaban por fuera del factor principal. En segundo lugar, los AFC realizados sobre los ítems conservados indicaron que todas las fortalezas poseen ajustes entre

\footnotetext{
${ }^{1}$ Expresión característica en el español rioplatense.
} 
Tabla 5. Estructura factorial basada en las correlaciones de Pearson

\begin{tabular}{|c|c|c|c|c|c|}
\hline \multirow{2}{*}{ Subescala } & \multicolumn{5}{|c|}{ Factor } \\
\hline & I & II & III & IV & $\mathrm{V}$ \\
\hline Liderazgo & .68 & & & & \\
\hline Valentía & .48 & & & & \\
\hline Perseverancia & .47 & & & & .31 \\
\hline Perspectiva & .46 & & & & \\
\hline Sentido del humor & .43 & & & .34 & \\
\hline Prudencia & & .75 & & & \\
\hline Autorregulación & & .54 & & & \\
\hline Integridad & & .53 & & & \\
\hline Equidad & & & -.64 & & \\
\hline Humildad & & & -.57 & & \\
\hline Trabajo en equipo & .39 & & -.53 & & \\
\hline Solidaridad & & & -.48 & & \\
\hline Capacidad de perdonar & & & -.44 & & \\
\hline Apertura mental & & .25 & -.29 & & \\
\hline Gratitud & & & & .72 & \\
\hline Capacidad de amar & & & & .65 & \\
\hline Vitalidad & & & & .46 & \\
\hline Optimismo & & & & .41 & .34 \\
\hline Religiosidad & & & & .35 & \\
\hline Amor al aprendizaje & & & & & .69 \\
\hline Curiosidad & & & & & .66 \\
\hline Creatividad & & & & & .52 \\
\hline Apreciación de la belleza & & & & & .30 \\
\hline Autovalor & 6.92 & 2.18 & 1.52 & 1.25 & 1.16 \\
\hline$\%$ de la varianza & 30.10 & 9.48 & 6.61 & 5.44 & 5.03 \\
\hline
\end{tabular}

excelentes y muy buenos ${ }^{2}$.

El AFE realizado con los puntajes totales de las fortalezas arrojó cinco factores sin forzar. La solución factorial resultante se presenta en la Tabla 5. La misma describió el $44.98 \%$ de la varianza de las puntuaciones.

A pesar de que el resultado obtenido es alentador pues coincide en gran medida con la propuesta de los autores, la literatura actual acerca del AFE desaconseja su uso con matrices de correlaciones de Pearson (utilizadas por defecto por el programa SPSS), cuando se trata de variables no normales o que tienen distribuciones asimétricas como en este caso (Ferrando \& Lorenzo-Seva, 2014; Lloret-Segura et al., 2014). Por lo tanto, se llevó a cabo un nuevo AFE con el programa FACTOR, que permite utilizar la matriz de correlaciones policóricas. Los factores se extrajeron mediante el Análisis Paralelo con implementación óptima (Timmerman \& Lorenzo-
Seva, 2011) y el método de mínimos cuadrados no ponderados (ULS).

Con respecto a la adecuación de la matriz, se obtuvo un determinante menor a .001 , el test de Bartlett fue significativo $(p<.001)$ y el KMO fue de .91 .

El análisis recomendó un solo factor, considerando el percentil 95 de la varianza. El GFI fue de .99, indicando un excelente ajuste, y el RMSEA de .03. La varianza explicada basada en los autovalores fue del 30\%. En la Tabla 6 se presentan los coeficientes de estructura y comunalidades de cada una de las fortalezas. Casi todas las fortalezas presentaron coeficientes por encima de 40 , salvo en las fortalezas de Integridad, Liderazgo y Autorregulación que obtuvieron coeficientes entre .21 y .31. Para la fortaleza de Prudencia, el coeficiente fue muy bajo y la comunalidad fue casi nula.

\footnotetext{
${ }^{2}$ Por falta de espacio, los valores de discriminación de cada ítem y los resultados de los AFE y AFC se pueden solicitar a los autores en el caso de que se los requiera. 
Tabla 6. Coeficientes de estructura y comunalidades de la estructura mediante Análisis Paralelo

\begin{tabular}{lcc}
\hline \multicolumn{1}{c}{ Fortaleza } & Coeficiente & Comunalidad \\
\hline Creatividad & .52 & .27 \\
Curiosidad & .53 & .30 \\
Apertura mental & .57 & .33 \\
Amor al aprendizaje & .56 & .31 \\
Perspectiva & .60 & .36 \\
Valentía & .62 & .38 \\
Perseverancia & .56 & .32 \\
Integridad & .30 & .09 \\
Vitalidad & .61 & .37 \\
Cap. de amar & .59 & .34 \\
Solidaridad & .66 & .43 \\
Trabajo en equipo & .64 & .40 \\
Equidad & .52 & .28 \\
Liderazgo & .32 & .10 \\
Capacidad de & .45 & .20 \\
perdonar & & \\
Humildad & .45 & .20 \\
Prudencia & .01 & .00 \\
Autorregulación & .21 & .05 \\
Gratitud & .45 & .20 \\
Apreciación de la & .74 & .55 \\
belleza & & \\
Optimismo & .63 & .40 \\
Sentido del humor & .57 & .32 \\
Religiosidad & .45 & .21 \\
\hline
\end{tabular}

\section{Fiabilidad}

Todas las subescalas que componen el instrumento presentaron coeficientes de consistencia interna por alfa de Cronbach de adecuados a muy buenos (entre .62 y .88) (ver Tabla 4). La escala de Gratitud presentó un índice con carencias $(\alpha=.55)$. Por último, la fortaleza de Inteligencia Social quedó compuesta sólo por tres ítems con una baja consistencia interna $(\alpha=.49)$ por lo que se decidió no utilizarla en los siguientes análisis.

\section{Diferencias en función del género, la edad y el tipo de escuela}

En la Tabla 7 se presenta el resultado de la comparación de cada una de las fortalezas en función del género, la edad y el tipo de escuela. Las comparaciones mostraron diferencias en función del género, a favor de las mujeres, en las fortalezas de Curiosidad, Perspectiva, Amor al aprendizaje, Integridad, Capacidad de amar, Solidaridad, Trabajo en equipo, Equidad, Humildad, Apreciación de la belleza, Gratitud y Religiosidad. En la única fortaleza en la que los varones mostraron mayores valores fue en
Autorregulación. En función de la edad, sólo se encontró que los adolescentes menores de 15 años, se perciben con mayor Capacidad de perdonar y Religiosidad que los más grandes. Por último, los adolescentes de escuela pública, se perciben con más Apertura mental, Amor al aprendizaje, Perseverancia, Trabajo en equipo, Equidad, Autorregulación y Optimismo, mientras que los adolescentes de escuelas privadas presentan puntajes significativamente más altos en Integridad, Prudencia y en Religiosidad.

\section{Correlaciones con otras pruebas}

En la Tabla 8 se muestran las correlaciones entre las fortalezas, los dominios del autoconcepto y la autoestima global. Se observó que la mayoría de las fortalezas se asocian a una autopercepción positiva. Específicamente, las fortalezas que más se asociaron a una autopercepción positiva fueron Vitalidad, Capacidad de amar, Liderazgo, Optimismo y Perspectiva. Estos resultados aportan evidencias a favor de la validez de constructo.

\section{Discusión}

El objetivo de este trabajo fue estudiar las propiedades psicométricas de la adaptación del Cuestionario de Fortalezas (VIA-Youth) para su uso con adolescentes de Argentina.

En primer lugar, se realizó la retro-traducción de la escala y se estudió la adecuación lingüística y conceptual a través de la evaluación de jueces expertos y una prueba piloto con adolescentes del medio local (población diana). Esta etapa aportó evidencias a favor de la validez de contenido. En segundo lugar, se analizó el funcionamiento de los ítems y la estructura interna de la escala mediante diferentes procedimientos. Se determinó la pertenencia de los ítems a cada una de las fortalezas mediante AFE y AFC. Por lo tanto, el ajuste del modelo de cada una de las fortalezas se obtuvo eliminando aquellos ítems que no se agrupaban en un único factor, los que presentaban bajas correlaciones ítem-total y los que bajaban la consistencia interna de la escala. Se logró entonces una estructura con menos ítems que el instrumento original. 
Tabla 7. Diferencias en función del género, la edad y el tipo de escuela a la que asiste el adolescente

\begin{tabular}{|c|c|c|c|c|c|c|c|c|c|c|c|c|}
\hline \multirow{3}{*}{ Fortalezas } & \multicolumn{4}{|c|}{ Género } & \multicolumn{4}{|c|}{ Edad } & \multicolumn{4}{|c|}{ Tipo de escuela } \\
\hline & $\begin{array}{l}\text { Varones } \\
(n=241)\end{array}$ & $\begin{array}{l}\text { Mujeres } \\
(n=296)\end{array}$ & $t$ & $r$ & $\begin{array}{c}\text { Menor } \\
15 \text { años } \\
(n=234)\end{array}$ & $\begin{array}{c}\text { Mayor } \\
15 \text { años } \\
(n= \\
303)\end{array}$ & $t$ & $r$ & $\begin{array}{l}\text { Escuela } \\
\text { pública } \\
(n=285)\end{array}$ & $\begin{array}{l}\text { Escuela } \\
\text { privada } \\
(n=252)\end{array}$ & $t$ & $r$ \\
\hline & $M(D T)$ & $M(D T)$ & & & $M(D T)$ & $M(D T)$ & & & $M(D T)$ & $M(D T)$ & & \\
\hline Creatividad & $\begin{array}{l}22.37 \\
(4.85)\end{array}$ & $\begin{array}{l}22.44 \\
(4.95)\end{array}$ & -0.16 & .01 & $\begin{array}{l}22.64 \\
(4.78)\end{array}$ & $\begin{array}{l}22.23 \\
(4.99)\end{array}$ & 0.96 & .07 & $\begin{array}{l}22.15 \\
(4.79)\end{array}$ & $\begin{array}{l}22.71 \\
(5.02)\end{array}$ & -1.30 & .10 \\
\hline Curiosidad & $\begin{array}{l}23.20 \\
(4.67)\end{array}$ & $\begin{array}{l}24.12 \\
(4.52)\end{array}$ & $-2.31 *$ & .18 & $\begin{array}{l}23.66 \\
(4.72)\end{array}$ & $\begin{array}{l}23.75 \\
(4.53)\end{array}$ & -0.21 & .02 & $\begin{array}{l}23.65 \\
(4.62)\end{array}$ & $\begin{array}{l}23.77 \\
(4.61)\end{array}$ & -0.29 & .02 \\
\hline Perspectiva & $\begin{array}{l}13.93 \\
(3.11)\end{array}$ & $\begin{array}{l}15.26 \\
(3.01)\end{array}$ & $\begin{array}{c}- \\
5.02 * * *\end{array}$ & .38 & $\begin{array}{l}14.71 \\
(3.23)\end{array}$ & $\begin{array}{l}14.63 \\
(3.04)\end{array}$ & $0.31^{\mathrm{a}}$ & .02 & $\begin{array}{l}14.71 \\
(3.22)\end{array}$ & $\begin{array}{l}14.61 \\
(3.01)\end{array}$ & 0.34 & .03 \\
\hline Valentía & $\begin{array}{l}22.96 \\
(4.15)\end{array}$ & $\begin{array}{l}23.08 \\
(4.40)\end{array}$ & -0.33 & .03 & $\begin{array}{l}22.91 \\
(4.61)\end{array}$ & $\begin{array}{l}23.11 \\
(4.02)\end{array}$ & -0.53 & .04 & $\begin{array}{l}23.32 \\
(4.18)\end{array}$ & $\begin{array}{l}22.70 \\
(4.38)\end{array}$ & 1.67 & .13 \\
\hline Apertura mental & $\begin{array}{l}11.61 \\
(2.51)\end{array}$ & $\begin{array}{l}12.01 \\
(2.66)\end{array}$ & -1.73 & .13 & $\begin{array}{l}11.63 \\
(2.68)\end{array}$ & $\begin{array}{l}11.99 \\
(2.53)\end{array}$ & -1.58 & .12 & $\begin{array}{l}12.14 \\
(2.40)\end{array}$ & $\begin{array}{l}11.48 \\
(2.77)\end{array}$ & $2.89^{\mathrm{a}} * *$ & .23 \\
\hline Amor al aprend. & $\begin{array}{l}19.05 \\
(4.14)\end{array}$ & $\begin{array}{l}19.85 \\
(3.86)\end{array}$ & $-2.30 * *$ & .18 & $\begin{array}{l}19.18 \\
(4.20)\end{array}$ & $\begin{array}{l}19.72 \\
(3.84)\end{array}$ & -1.55 & .12 & $\begin{array}{l}20.24 \\
(3.73)\end{array}$ & $\begin{array}{l}18.64 \\
(4.14)\end{array}$ & $4.68^{\mathrm{a} * * *}$ & .36 \\
\hline Perseverancia & $\begin{array}{l}18.39 \\
(3.68)\end{array}$ & $\begin{array}{l}18.65 \\
(3.59)\end{array}$ & -0.80 & .06 & $\begin{array}{l}18.70 \\
(3.54)\end{array}$ & $\begin{array}{l}18.41 \\
(3.71)\end{array}$ & 0.91 & .07 & $\begin{array}{l}18.83 \\
(3.65)\end{array}$ & $\begin{array}{l}18.20 \\
(3.60)\end{array}$ & $2.01 *$ & .15 \\
\hline Integridad & $\begin{array}{l}12.04 \\
(3.79)\end{array}$ & $\begin{array}{l}12.72 \\
(3.69)\end{array}$ & $-2.10^{*}$ & .16 & $\begin{array}{l}12.14 \\
(3.74)\end{array}$ & $\begin{array}{l}12.62 \\
(3.74)\end{array}$ & -1.47 & .11 & $\begin{array}{l}12.11 \\
(3.82)\end{array}$ & $\begin{array}{l}12.75 \\
(3.64)\end{array}$ & $-1.98 *$ & .15 \\
\hline Vitalidad & $\begin{array}{l}18.96 \\
(3.51)\end{array}$ & $\begin{array}{l}19.60 \\
(4.03)\end{array}$ & $-1.95^{\mathrm{a}}$ & .15 & $\begin{array}{l}19.64 \\
(3.69)\end{array}$ & $\begin{array}{l}19.05 \\
(3.90)\end{array}$ & 1.76 & .13 & $\begin{array}{l}19.50 \\
(3.83)\end{array}$ & $\begin{array}{l}19.09 \\
(3.80)\end{array}$ & 1.23 & .09 \\
\hline Cap. de amar & $\begin{array}{l}15.04 \\
(3.53)\end{array}$ & $\begin{array}{l}15.92 \\
(3.21)\end{array}$ & $-2.98^{a} * *$ & .24 & $\begin{array}{l}15.71 \\
(3.41)\end{array}$ & $\begin{array}{l}15.37 \\
(3.37)\end{array}$ & 1.14 & .09 & $\begin{array}{l}15.42 \\
(3.46)\end{array}$ & $\begin{array}{l}15.64 \\
(3.30)\end{array}$ & -0.74 & .06 \\
\hline Solidaridad & $\begin{array}{l}19.29 \\
(3.72)\end{array}$ & $\begin{array}{l}21.05 \\
(3.29)\end{array}$ & $5.83^{-} * * *$ & .43 & $\begin{array}{l}20.34 \\
(3.59)\end{array}$ & $\begin{array}{l}20.20 \\
(3.60)\end{array}$ & 0.46 & .04 & $\begin{array}{l}20.53 \\
(3.34)\end{array}$ & $\begin{array}{l}19.95 \\
(3.84)\end{array}$ & 1.87 & .14 \\
\hline Trab. en equipo & $\begin{array}{l}23.28 \\
(4.23)\end{array}$ & $\begin{array}{l}24.25 \\
(3.99)\end{array}$ & $-2.72 * *$ & .21 & $\begin{array}{l}23.91 \\
(4.03)\end{array}$ & $\begin{array}{l}23.74 \\
(4.20)\end{array}$ & 0.46 & .04 & $\begin{array}{l}24.15 \\
(4.12)\end{array}$ & $\begin{array}{l}23.43 \\
(4.09)\end{array}$ & $2.00 *$ & .15 \\
\hline Equidad & $\begin{array}{l}21.35 \\
(4.68)\end{array}$ & $\begin{array}{l}22.53 \\
(4.53)\end{array}$ & $-2.96 * *$ & .23 & $\begin{array}{l}22.21 \\
(4.75)\end{array}$ & $\begin{array}{l}21.84 \\
(4.54)\end{array}$ & 0.93 & .07 & $\begin{array}{l}22.72 \\
(4.66)\end{array}$ & $\begin{array}{l}21.19 \\
(4.47)\end{array}$ & $3.87 * * *$ & .29 \\
\hline Liderazgo & $\begin{array}{l}18.74 \\
(4.74)\end{array}$ & $\begin{array}{l}19.08 \\
(4.69)\end{array}$ & -0.81 & .06 & $\begin{array}{l}18.76 \\
(4.80)\end{array}$ & $\begin{array}{l}19.06 \\
(4.65)\end{array}$ & -0.73 & .06 & $\begin{array}{l}18.95 \\
(4.69)\end{array}$ & $\begin{array}{l}18.90 \\
(4.75)\end{array}$ & 0.14 & .01 \\
\hline $\begin{array}{l}\text { Cap. de } \\
\text { perdonar }\end{array}$ & $\begin{array}{l}14.55 \\
(3.73)\end{array}$ & $\begin{array}{l}14.85 \\
(3.87)\end{array}$ & -0.92 & .07 & $\begin{array}{l}15.09 \\
(3.84)\end{array}$ & $\begin{array}{l}14.43 \\
(3.77)\end{array}$ & $1.99 *$ & .15 & $\begin{array}{l}14.59 \\
(3.91)\end{array}$ & $\begin{array}{l}14.86 \\
(3.70)\end{array}$ & -0.80 & .06 \\
\hline Humildad & $\begin{array}{l}26.05 \\
(4.83)\end{array}$ & $\begin{array}{l}27.40 \\
(4.83)\end{array}$ & $-3.21 * *$ & .24 & $\begin{array}{l}26.88 \\
(4.60)\end{array}$ & $\begin{array}{l}26.72 \\
(5.08)\end{array}$ & 0.38 & .03 & $\begin{array}{l}27.17 \\
(4.69)\end{array}$ & $\begin{array}{l}26.36 \\
(5.04)\end{array}$ & 1.91 & .15 \\
\hline Prudencia & $\begin{array}{l}7.05 \\
(2.79)\end{array}$ & $\begin{array}{c}6.92 \\
(2.71)\end{array}$ & 0.52 & .04 & $\begin{array}{c}6.83 \\
(2.72)\end{array}$ & $\begin{array}{l}7.09 \\
(2.77)\end{array}$ & -1.09 & .08 & $\begin{array}{c}6.74 \\
(2.72)\end{array}$ & $\begin{array}{c}7.25 \\
(2.76)\end{array}$ & $-2.13 *$ & .16 \\
\hline Autorregulación & $\begin{array}{l}18.44 \\
(5.46)\end{array}$ & $\begin{array}{l}16.75 \\
(5.75)\end{array}$ & $3.47 * *$ & .26 & $\begin{array}{l}17.57 \\
(5.51)\end{array}$ & $\begin{array}{l}17.46 \\
(5.82)\end{array}$ & 0.23 & .02 & $\begin{array}{l}18.23 \\
(5.69)\end{array}$ & $\begin{array}{l}16.69 \\
(5.57)\end{array}$ & $3.16 * *$ & .24 \\
\hline $\begin{array}{l}\text { Ap. de la } \\
\text { belleza }\end{array}$ & $\begin{array}{l}16.29 \\
(4.98)\end{array}$ & $\begin{array}{l}19.67 \\
(4.43)\end{array}$ & 8. $32^{-} * * *$ & .60 & $\begin{array}{l}18.06 \\
(5.26)\end{array}$ & $\begin{array}{l}18.22 \\
(4.75)\end{array}$ & -0.36 & .03 & $\begin{array}{l}18.18 \\
(4.61)\end{array}$ & $\begin{array}{l}18.12 \\
(5.36)\end{array}$ & $0.14^{\mathrm{a}}$ & .01 \\
\hline Gratitud & $\begin{array}{l}17.15 \\
(2.47)\end{array}$ & $\begin{array}{l}17.67 \\
(2.32)\end{array}$ & $-2.51 * *$ & .19 & $\begin{array}{l}17.34 \\
(2.58)\end{array}$ & $\begin{array}{l}17.51 \\
(2.26)\end{array}$ & $-0.79^{\mathrm{a}}$ & .06 & $\begin{array}{l}17.56 \\
(2.31)\end{array}$ & $\begin{array}{l}17.30 \\
(2.50)\end{array}$ & 1.26 & .10 \\
\hline Optimismo & $\begin{array}{l}19.51 \\
(4.02)\end{array}$ & $\begin{array}{l}19.44 \\
(4.35)\end{array}$ & 0.18 & .01 & $\begin{array}{l}19.43 \\
(3.98)\end{array}$ & $\begin{array}{l}19.50 \\
(4.37)\end{array}$ & -0.19 & .02 & $\begin{array}{l}19.82 \\
(4.11)\end{array}$ & $\begin{array}{l}19.07 \\
(4.28)\end{array}$ & $2.06^{*}$ & .16 \\
\hline Sent. del humor & $\begin{array}{l}15.65 \\
(3.58)\end{array}$ & $\begin{array}{l}15.93 \\
(3.38)\end{array}$ & -0.90 & .07 & $\begin{array}{l}15.85 \\
(3.57)\end{array}$ & $\begin{array}{l}15.77 \\
(3.40)\end{array}$ & 0.23 & .02 & $\begin{array}{l}15.85 \\
(3.62)\end{array}$ & $\begin{array}{l}15.75 \\
(3.30)\end{array}$ & 0.32 & .02 \\
\hline Religiosidad & $\begin{array}{l}20.92 \\
(6.83)\end{array}$ & $\begin{array}{l}22.66 \\
(6.16)\end{array}$ & $\begin{array}{c}- \\
3.09 * * *\end{array}$ & .23 & $\begin{array}{l}22.72 \\
(5.71)\end{array}$ & $\begin{array}{l}21.23 \\
(7.02)\end{array}$ & $2.70^{\mathrm{a} * *}$ & .21 & $\begin{array}{l}21.35 \\
(6.73)\end{array}$ & $\begin{array}{l}22.48 \\
(6.23)\end{array}$ & $-2.01^{\mathrm{a} *}$ & .15 \\
\hline
\end{tabular}

Nota: $r=$ tamaño del efecto.

${ }^{a}$ Por no cumplirse la igualdad de varianzas según la Prueba de Levene, se utilizó la prueba $t$ para varianzas desiguales. $* p<.05 * * p<.01 * * * p<.001$ (bilateral) 
Tabla 8. Correlaciones entre las fortalezas y las autopercepciones de los adolescentes

\begin{tabular}{|c|c|c|c|c|c|c|c|c|c|}
\hline Fortalezas & $\begin{array}{c}\text { Competencias } \\
\text { académicas }\end{array}$ & $\begin{array}{c}\text { Aceptación } \\
\text { social }\end{array}$ & $\begin{array}{c}\text { Competencias } \\
\text { deportivas }\end{array}$ & $\begin{array}{c}\text { Aparienci } \\
\text { física }\end{array}$ & $\begin{array}{c}\text { LaCompetencia } \\
\text { Laboral }\end{array}$ & $\begin{array}{l}\text { Atractivo } \\
\text { amoroso }\end{array}$ & $\begin{array}{c}\text { Buen } \\
\text { comportamiento }\end{array}$ & $\begin{array}{l}\text { Amistad } \\
\text { íntima }\end{array}$ & $\begin{array}{c}\text { Autoestima } \\
\text { global }\end{array}$ \\
\hline Creatividad & .21 & $.31^{* *}$ & .07 & .11 & $.24^{*}$ & 08 & $.34^{* *}$ & .04 & .12 \\
\hline Curiosidad & $.27^{*}$ & .02 & .01 & .01 & .07 & -.05 & $.25^{*}$ & .02 & .05 \\
\hline Apertura mental & .07 & $.28^{*}$ & .05 & -.03 & .11 & $.27^{*}$ & .13 & $.28^{*}$ & -.12 \\
\hline Amor al aprend. & .03 & $.53^{* *}$ & .09 & $.25^{*}$ & -05 & $.32^{* *}$ & .01 & $.38^{* *}$ & .21 \\
\hline Perspectiva & $.32^{* *}$ & $.38^{* *}$ & $.35^{* *}$ & .10 & $.32^{* *}$ & .23 & $.39^{* * *}$ & .03 & .19 \\
\hline Valentía & $.30^{*}$ & -.06 & .18 & -.16 & .14 & .04 & $.32^{* *}$ & -.12 & -.17 \\
\hline Perseverancia & $.29^{*}$ & .16 & .18 & .08 & $.25^{*}$ & .20 & $.25^{*}$ & -.01 & .10 \\
\hline Integridad & $.28^{*}$ & 22 & -.00 & .17 & -.08 & .02 & $.31^{* * *}$ & .17 & .17 \\
\hline Vitalidad & .12 & $.49^{* *}$ & .10 & $.36^{* *}$ & .22 & $.44^{* *}$ & $.31^{* * *}$ & .20 & $.50^{* * *}$ \\
\hline Cap. de amar & -.01 & $.37^{* *}$ & .11 & $.28^{*}$ & .10 & $.41^{* *}$ & .14 & $.37^{* *}$ & .19 \\
\hline Solidaridad & -.07 & .12 & .01 & -.14 & .09 & .18 & .10 & .20 & $-.27^{*}$ \\
\hline Trab. en equipo & .08 & $.25^{*}$ & $.26^{*}$ & .10 & .22 & $.35^{* *}$ & $.37^{* *}$ & .05 & .06 \\
\hline Equidad & .02 & .01 & .03 & -.01 & .03 & .02 & $.40^{* * *}$ & -.20 & -.09 \\
\hline Liderazgo & $.51^{* *}$ & $.36^{* *}$ & $.25^{*}$ & .09 & $.46^{* *}$ & $.31^{* *}$ & $.34^{* *}$ & .13 & .21 \\
\hline $\begin{array}{l}\text { Cap. de } \\
\text { perdonar }\end{array}$ & -.01 & .17 & .01 & .17 & -.05 & .07 & .01 & -.03 & .03 \\
\hline Humildad & -.16 & -.13 & .02 & $-.32^{* *}$ & -.06 & -.16 & .07 & -.13 & $-.33^{* *}$ \\
\hline Prudencia & .21 & -.05 & -.03 & -.12 & .19 & -.18 & .15 & -.21 & -.08 \\
\hline Autorregulación & .15 & -.08 & .13 & .05 & -.06 & -.15 & $.26^{*}$ & $-.37^{* *}$ & .10 \\
\hline Gratitud & .06 & .03 & -.07 & -.14 & .01 & .02 & -.01 & .16 & -.18 \\
\hline $\begin{array}{l}\text { Ap. de la } \\
\text { belleza }\end{array}$ & .01 & 08 & -.04 & .21 & .02 & $.33^{* *}$ & $.24^{*}$ & .08 & .22 \\
\hline Optimismo & .19 & $.38^{* *}$ & $.30^{*}$ & $.39^{* *}$ & $.24^{*}$ & $.47^{* *}$ & $.35^{* *}$ & .19 & $.48^{* *}$ \\
\hline Sent. del humor & -.11 & $.29^{*}$ & .06 & .01 & .08 & .11 & -.04 & .11 & .02 \\
\hline Religiosidad & .12 & .01 & .12 & .08 & -.04 & $.35^{* *}$ & .08 & .21 & .04 \\
\hline
\end{tabular}

$* p<.05 . * * p<.01 . * * * p<.001$ (bilateral)

Los AFE realizados sobre las fortalezas indicaron que la estructura interna variaba en función del tipo de análisis y la matriz de correlaciones sobre la que se realiza. En el caso de buscar consistencia con la versión norteamericana (Park \& Peterson, 2006), la alemana (Ruch et al., 2014), la australiana (Toner et al., 2012) e incluso la española (Giménez, 2010), el primer AFE realizado presentaría coherencia con los anteriores estudios debido a que se obtuvieron cinco factores que agrupaban fortalezas con un contenido esperable.

Por ejemplo, el Factor I quedó compuesto por las fortalezas de Liderazgo, Valentía, Sentido del humor, Perspectiva y Perseverancia, que puede implicar la fuerza necesaria para persistir tras una meta, reflejando el ejercicio consciente de la voluntad hacia objetivos que muchas veces no se sabe si se podrán alcanzar. A su vez, implica una apertura en el modo de interpretar el mundo y hacia los demás. La Templanza (Factor II) implica la expresión moderada de apetitos y necesidades, mediante la cual la persona no reprime sus aspiraciones pero espera la oportunidad de satisfacerlas sin perjudicar a nadie. Humanidad y justicia (Factor III) trata de un conjunto de fortalezas sociales que implican empatía, compasión y generosidad, necesarias para convivir con otros. Se incluye la apertura mental, que supone la capacidad de pensar las situaciones desde distintas perspectivas, necesaria para llegar a acuerdos con los demás. El Factor IV, Trascendencia, comprende un conjunto de fortalezas que forjan las conexiones con un universo más amplio y le dan sentido a la vida. Por último, el Factor $\mathrm{V}$ de Sabiduría y Conocimiento refiere a la predisposición para aprender, conocer y pensar nuevas formas de hacer las cosas y apreciar la belleza del mundo.

Sin embargo, las recomendaciones actuales sobre el AFE desaconsejan enérgica y explícitamente el uso del método de componentes principales para la estimación de los factores (siendo el método utilizado en todas las adaptaciones del VIA-Youth). En su lugar recomiendan trabajar, en primera instancia, con las matrices policóricas cuando se trata de variables ordinales con distribuciones no normales $\mathrm{y}$, en segundo lugar, estimar los factores mediante AP y el método ULS (Ferrando \& Lorenzo-Seva, 2014; Lloret-Segura et al., 2014). Aunque el 
primer AFE se realizó extrayendo los factores por el método de máxima verosimilitud, persistía el problema de la utilización de las matrices de correlaciones de Pearson.

Por lo tanto, el análisis realizado mediante estas recomendaciones sugiere un solo factor que agrupa a todas las fortalezas. Salvo las fortalezas de Integridad, Liderazgo y Autorregulación, todas presentaron pesajes por encima de 40 . Para la fortaleza de Prudencia, el peso fue muy bajo y la comunalidad resultó casi nula.

Peterson y Seligman (2004) observaron que las fortalezas de templanza (e.g. autorregulación, prudencia, humildad, integridad) eran las más difíciles de medir, y a lo largo de las adaptaciones, fueron las que obtuvieron los coeficientes de consistencia interna más bajos (Giménez, 2010; Toner et al., 2012; Van Eeden et al., 2008). Asimismo, se trata de las escalas que presentan, tanto en adolescentes argentinos como en los de otras poblaciones, los puntajes medios más bajos, por lo que habría que indagar si se trata de aspectos positivos evaluables en esta etapa vital (Van Eeden et al., 2008).

Con respecto a la fortaleza de Inteligencia Social, se decidió eliminarla, debido a su baja consistencia interna. En la versión española este índice también fue inaceptable (Giménez, 2010). Se trata de constructo complejo, con un vasto desarrollo teórico en nuestra disciplina y en función de los análisis se pudo reparar que los ítems de esta escala no son un buen reflejo de ello. En futuras versiones de la escala será importante generar ítems que puedan evaluar esta fortaleza de manera válida y fiable.

La adaptación sudafricana (Van Eeden et al., 2008) sugiere que la estructura del VIA-Youth es unidimensional. Se trata del único estudio hasta el momento que analiza la agrupación de las fortalezas a través de diferentes procedimientos (AFE y AFC). En el presente trabajo, también se probaron diferentes métodos y se arribó a la misma conclusión: la estructura propuesta desde la teoría no se replica en los datos, al menos con los análisis aconsejados por la literatura actual. A pesar de ello, se puede hacer uso de las fortalezas por separado, ya que el proceso de validación mostrado en esta adaptación indica que cada escala posee adecuadas cualidades psicométricas $\mathrm{y}$ de este modo no se pierde riqueza en su evaluación. Podría argumentarse, en última instancia, que han sido los mismos autores de la clasificación de las fortalezas humanas (Peterson $\&$ Seligman, 2004) los que han postulado que para captar en su totalidad la noción del "buen carácter" se propone una clasificación de carácter plural, que implica un conjunto de virtudes y fortalezas.

Los resultados han mostrado, una y otra vez, que las puntuaciones obtenidas por los adolescentes apuntan hacia una dirección positiva, es decir, en general los jóvenes desarrollan la mayoría de estas características positivas (Park \& Peterson, 2003).

Con respecto al perfil de fortalezas de los adolescentes del presente trabajo, se mostró que las fortalezas más destacadas son: solidaridad, trabajo en equipo y sentido del humor. En general, los diferentes estudios muestran que los adolescentes tienen medias especialmente altas en las fortalezas humanitarias y especialmente bajas en las de templanza. Las fortalezas más comunes entre los adolescentes de diferentes culturas son: gratitud, sentido del humor y capacidad de amar y las menos comunes: prudencia, capacidad de perdonar, religiosidad y autorregulación (Giménez, 2010; Park \& Peterson, 2003; Ruch et al., 2014).

Al analizar diferencias entre géneros, se encontró que las jóvenes puntúan más alto en algunas fortalezas como apreciación de la belleza, equidad, generosidad y perspectiva y este resultado se mantiene en los otros estudios con adolescentes de las demás poblaciones (Giménez, 2010; Park \& Peterson, 2006; Ruch et al., 2014; Toner et al., 2012).

En cuanto a diferencias en función de la edad los estudios muestran que los menores puntúan más alto que los mayores en muchas de las fortalezas (Giménez, 2010; Park \& Peterson, 2006; Ruch et al., 2014). En el presente trabajo se encontró sólo diferencias significativas en la capacidad de perdonar y en la religiosidad, aunque en general presentaron puntajes más altos.

Con respecto al tipo de escuela a la que asisten, se encontró que los adolescentes de escuela pública se perciben significativamente con más apertura mental, perseverancia y optimismo, entre otras fortalezas, mientras los de escuela privada presentaron puntajes significativamente 
más altos en integridad, prudencia y religiosidad. Es esperable que, en función a los diferentes contextos que rodean a los adolescentes (padres y escuela), se encuentren perfiles de fortalezas específicos. El hecho de que las escuelas privadas participantes fueran religiosas puede tener que ver con estos resultados. Los trabajos en donde se comparan las fortalezas en función de los grupos socio-culturales encuentran, por ejemplo, que los afro-americanos y las personas de raza negra en Sudáfrica se perciben más religiosos que los adolescentes de otros grupos (Park \& Peterson, 2006; Van Eeden et al., 2008). Los valores y las prácticas sociales que circulan en los contextos en donde viven y se desarrollan los adolescentes puede influir en la percepción de sus fortalezas humanas.

Por último, como evidencias adicionales a la validez de constructo, se encontró que en general las fortalezas se asocian a una autopercepción positiva y, por lo tanto a un desarrollo positivo en los adolescentes. El autoconcepto en sus diferentes dominios específicos es un aspecto sumamente relevante en el desarrollo de niños y adolescentes (Molina, Raimundi, López, Cataldi, \& Bugallo, 2011; Schmidt, Messoulam, \& Molina, 2008). Diversos trabajos han estudiado las relaciones de las fortalezas con diferentes indicadores de ajuste psicosocial y bienestar (e.g. Gillham et al., 2011; Proyer et al., 2012) mostrando que se trata de características personales asociadas a un desarrollo saludable. En adolescentes españoles (Giménez, 2010) también se encontró que la correlación más fuerte con autoestima global se da con las fortalezas de optimismo y vitalidad.

\section{Limitaciones y direcciones futuras}

Estos resultados corresponden a una primera etapa en la validación de este instrumento. Su contribución es doble: aporta datos acerca de las cualidades psicométricas de la adaptación lograda y pone en evidencia la importancia de considerar las estrategias de análisis factoriales utilizadas. Evidentemente es necesario considerar los métodos con los cuales se realiza el análisis de la estructura interna de la escala porque, a partir de ellos, los resultados varían de modo tal que tienen implicancias sobre la evaluación de este constructo.
En un futuro, por un lado, será necesario factorizar todos los ítems del cuestionario, evitando el uso de parcels, para poder estudiar el funcionamiento de la escala de forma global. Por el otro, esta adaptación presentó 115 ítems, en lugar de los 198 de la versión original, debido a los criterios de selección utilizados. Sin embargo resultó un instrumento demasiado largo para su administración con adolescentes. Es por ello que, para lograr un instrumento más breve, podría ser interesante seleccionar aquellos ítems que tengan un mejor funcionamiento (mediante criterios empíricos) y de esta manera lograr un instrumento válido y fiable, con mayor utilidad para su evaluación en población adolescente.

Asimismo, existen dificultades métricas respecto de la fortaleza de Inteligencia social por lo que es necesario revisar su contenido para tratar de elaborar indicadores culturalmente relevantes.

La escala fue aplicada a adolescentes de cuatro escuelas de la Ciudad de Buenos Aires. Aunque se trata de una muestra heterogénea, que contempla adolescentes con características sociodemográficas diversas será necesario seguir estudiando la validez y fiabilidad de la escala con adolescentes de otros contextos de Argentina, ya que se trata de un país con un territorio extenso y una diversidad sociocultural importante.

A su vez, será necesario indagar la relación con criterios externos que sean ejemplos empíricos del comportamiento de los adolescentes (e.g. experiencias positivas en diferentes actividades o rendimiento académico) para estudiar la validez orientada al criterio.

El Inventario de Fortalezas para adolescentes ha demostrado ser útil en diferentes países para evaluar un constructo novedoso en un creciente enfoque en nuestra disciplina. Poder contar con una adaptación válida para su uso con adolescentes de Argentina, implica no sólo la posibilidad de comparar estos resultados con los realizados en otros países, sino contar con una herramienta útil para el trabajo de los profesionales de la salud en el desarrollo positivo de los adolescentes.

Esta adaptación implicó una primera aproximación a la evaluación y el estudio de este constructo complejo en nuestro contexto sociocultural. Aun así, el instrumento resultó ser 
válido y fiable para la medición las diferentes fortalezas humanas en los adolescentes.

\section{Referencias}

Arias, B. (2008). Desarrollo de un ejemplo de análisis factorial confirmatorio con LISREL, AMOS y SAS. Seminario de actualización en Investigación sobre Discapacidad SAID. Universidad de Valladolid. Recuperado de http://www.benitoarias.com/articulos/afc.pdf

Balaguer, I., Castillo, I., \& Duda, J. (2008). Apoyo a la autonomía, satisfacción de las necesidades, motivación y bienestar en deportistas de competición: Un análisis de la teoría de la autodeterminación. Revista de Psicología del Deporte, 17(1), 123-139.

Bassi, M., Steca, P., Monzani, D., Greco, A., \& Delle Fave, A. (2013). Personality and optimal experience in adolescence: Implications for well-being and development. Journal of Happiness Studies, 14(3), 1-15. doi:10.1007/s10902-013-9451-x

Brdar, I., \& Kashdan, T. B. (2010). Character strengths and well-being in Croatia: An empirical investigation of structure and correlates. Journal of Research in Personality, 44(1), 151-154. doi:10.1016/j.jrp.2009.12.001

Cohen, J. (1992). A power primer. Psychological Bulletin, 112(1), 155-159. doi:10.1037/00332909.112.1.155

Ferragut, M., Blanca, M. J., \& Ortiz-Tallo, M. (2013). Psychological values as protective factors against sexist attitudes in preadolescents. Psicothema, 25(1), 38-42. doi: $10.7334 /$ psicothema2012.85

Ferragut, M., Blanca, M. J., \& Ortiz-Tallo, M. (2014). Psychological virtues during adolescence: A longitudinal study of gender differences. European Journal of Developmental Psychology, 11(5), 521-531. doi:10.1080/17405629.2013.876403

Ferrando, P., \& Lorenzo-Seva, U. (2014). El análisis factorial exploratorio de los ítems: Algunas consideraciones adicionales. Anales de Psicología, 30(3), 1170-1175. doi:dx.doi.org/10.6018/analesps.30.3.199991

Gillham, J., Adams-Deutsch, Z., Werner, J., Reivich, K., Coulter-Heindl, V., Linkins, M., ... Seligman, M. (2011). Character strengths predict subjective well-being during adolescence. The Journal of Positive Psychology, 6(1), 31-44. doi:10.1080/174397 60.2010 .536773

Giménez, M. (2010). La medida de las fortalezas psicológicas en adolescentes (VIA-Youth): Relación con clima familiar, psicopatología y bienestar psicológico. Tesis doctoral. Universidad Complutense de Madrid. Recuperado de http://eprints.ucm.es/11578/1/ T32253.pdf?origin=publication_detail

Grinhauz, A., \& Castro Solano, A. (2014). La evaluación de las virtudes y fortalezas del carácter en niños argentinos: Adaptación y validación del Inventario de Virtudes y Fortalezas para niños. Summa Psicológica UST, 11(1), 115-126.

Khumalo, I., Wissing, M., \& Temane, Q. (2008). Exploring the validity of the Values-In-Action Inventory of Strengths (VIA-IS) in an African context. Journal of Psychology in Africa, 18(February 2015), 133-142. doi:10.1080/14330237.2008.10820180

Linley, P., Maltby, J., Wood, A., Joseph, S., Harrington, S., Peterson, C., ... Seligman, M. (2007). Character strengths in the United Kingdom: The VIA Inventory of Strengths. Personality and Individual Differences, 43(2), 341-351. doi:10.1016/j.paid.2006.12.004

Littman-Ovadia, H., \& Lavy, S. (2012). Character Strengths in Israel. European Journal of Psychological Assessment, 28(1), 41-50. doi:10.1027/1015-5759/a000089

Lloret-Segura, S., Ferreres-Traver, A., HernándezBaeza, A., \& Tomás-Marco, I. (2014). El análisis factorial exploratorio de los ítems: una guía práctica, revisada y actualizada. Anales de Psicología, 30(3), 1151-1169. doi:10.6018/analesps.30.3.199361

Lorenzo-Seva, U., \& Ferrando, P. (2015). FACTOR: A computer program to fit the exploratory factor analysis model. University Rovira i Virgili.

Lounsbury, J., Fisher, L., Levy, J., \& Welsh, D. (2009). An investigation of character strengths in relation to the academic success of college students. Individual Differences Research, 7(1), 52-69. Recuperado de http://h24files.s3.amazonaws.com/80877/189235Xtfd6.pdf 
Molina, M. F., Raimundi, M. J., López, C., Cataldi, S., \& Bugallo, L. (2011). Adaptación del Perfil de Autopercepciones para niños para su uso en la Ciudad de Buenos Aires. Revista Iberoamericana de Diagnóstico y Evaluación - e Avaliação Psicológica, 2(32), 53-78.

Niemiec, R. (2013). VIA Character strenghts: Research and practice (the first 10 years). En H. Knoop \& A. Delle Fave (Eds.), Well-being and cultures (pp. 11-30). New York: Springer.

Park, N., \& Peterson, C. (2003). Assesment of character strengths among youth: The values in Action Inventory of Strenghts for Youth. Indicators of Positive Developtment. Recuperado de http://www.childtrends.org/Fi les/Child_Trends-2003_03_12_PD_PDConf PetersonPark.pdf

Park, N., \& Peterson, C. (2006). Moral competence and character strengths among adolescents: The development and validation of the Values in Action Inventory of Strengths for Youth. Journal of Adolescence, 29(6), 891-909. doi:10.1016/j.adolescence.2006.04. 011

Peterson, C., \& Seligman, M. (2004). Character strengths and virtues: A handbook and classification. New York: Oxford University Press.

Proyer, R., Sidler, N., Weber, M., \& Ruch, W. (2012). A multi-method approach to studying the relationship between character strengths and vocational interests in adolescents. International Journal for Educational and Vocational Guidance, 12(2), 141-157.

Ruch, W., Weber, M., Park, N., \& Peterson, C. (2014). Character strengths in children and adolescents. Reliability and initial validity of the German Values in Action Inventory of Strenghts for Youth (German VIA-Youth). European Journal of Psychological Assessment, 30(1), 57-64. doi:10.1027/10155759/a000169

Schmidt, V., Messoulam, N., \& Molina, M. F. (2008). Autoconcepto académico en adolescentes de escuelas medias: Presentación de un instrumento para su evaluación. Revista Iberoamericana de Diagnóstico y Evaluación - e Avaliação Psicológica, 1, 81-106.
Seligman, M., \& Csikszentmihalyi, M. (2000). Positive psychology: An introduction. American Psychologist, 55(1), 5-14. doi:10.1037//0003-066X.55.1.5

Shimai, S., Otake, K., Park, N., Peterson, C., \& Seligman, M. (2006). Convergence of character strengths in american and japanese young adults. Journal of Happiness Studies, 7(3), 311-322. doi:10.1007/s10902-005-36477

Steen, T., Kachorek, L., \& Peterson, C. (2003). Character strengths among youth. Journal of Youth and Adolescence, 32(1), 5-16. Recuperado a partir de http://link.springer.com/article/10.1023/A:102 1024205483

Timmerman, M., \& Lorenzo-Seva, U. (2011). Dimensionality assessment of ordered polytomous items with parallel analysis. Psychological Methods, 16, 209-220.

Toner, E., Haslam, N., Robinson, J., \& Williams, P. (2012). Character strengths and wellbeing in adolescence: Structure and correlates of the Values in Action Inventory of Strengths for Children. Personality and Individual Differences, 52(5), 637-642. doi:10.1016/j.pa id.2011.12.014

Van Eeden, C., Wissing, M., Dreyer, J., Park, N., \& Peterson, C. (2008). Validation of the Values in Action Inventory of Strengths for Youth (VIA-Youth) among South African learners. Journal of Psychology in Africa, 18, 143-154. doi:10.1080/14330237.2008.10820 181

Weber, M., Ruch, W., Littman-Ovadia, H., Lavy, S., \& Gai, O. (2013). Relationships among higher-order strengths factors, subjective well-being, and general self-efficacy - The case of Israeli adolescents. Personality and Individual Differences, 55(3), 322-327. doi:10.1016/j.paid.2013.03.006 